\title{
Una nueva especie de Miconia (Melastomataceae) de los bosques altoandinos de Bogotá D.C., Colombia
}

\author{
A new species of Miconia (Melastomataceae) from the \\ high Andean forest of Bogotá D.C., Colombia
}

\section{Acta Botanica Mexicana}

\author{
Humberto Mendoza-Cifuentes ${ }^{1,2}$ (D)
}

\section{Resumen:}

Antecedentes y Objetivos: Miconia, con cerca de 1900 especies, es el género de las angiospermas leñosas más diverso en el Neotrópico y uno de los más grandes del planeta. Durante el desarrollo del proyecto Flora de Bogotá, se encontró una especie no descrita de Miconia, única entre sus congéneres en caracteres de hábito de crecimiento y tipo de tricomas. El objetivo de este artículo es describir esta nueva especie hasta ahora conocida solo de los alrededores del Distrito Capital y compararla con los taxones más similares morfológicamente.

Métodos: Se realizó la descripción e ilustración de la nueva especie a partir de colecciones depositadas en el herbario JBB del Jardín Botánico de Bogotá. Las medidas de las partes vegetativas e inflorescencia se realizaron a partir de material seco, mientras que las estructuras florales y frutos fueron medidas a partir de material rehidratado. Se sugiere su estado de conservación con base en los criterios de la IUCN.

Resultados clave: Miconia mahechae sp. nov. se caracteriza por su hábito arbustivo-lianescente, entrenudos oblongos, indumento denso-lanoso compuesto de tricomas alargado-irregulares rugosos en entrenudos, peciolos e inflorescencia, hojas ovado a elípticas, 3-nervadas, con la haz lisa y ápice obtuso o agudo, bractéolas inconspicuas, anteras oblongo-obovadas con un poro apical y conectivo basal sin prolongaciones.

Conclusiones: La especie nueva se relaciona morfológicamente con Miconia ruizteranii, de la cual se distingue por la forma de las anteras y el indumento de tallos e inflorescencia. Hasta el momento, M. mahechae solo se conoce de los alrededores de la ciudad de Bogotá y los Parques Nacionales Chingaza y Sumapaz, en zonas de bosque altoandino. Con base en los criterios de la IUCN se sugiere su categorización como Vulnerable (VU).

Palabras clave: bosque altoandino, Miconieae, norte de los Andes, taxonomía.

\section{Abstract:}

Background and Aims: Miconia, with over 1900 species, is the largest exclusively Neotropical genus of woody vascular plants and one of the largest among flowering plants globally. During the development of the Flora de Bogotá project, an undescribed species of Miconia, unique among its congeners in terms of growth habit and trichome type, was found. The aim of this paper is to describe this new species so far known only from the surroundings of the Capital District and to compare it with the most morphologically similar taxa.

Methods: The description and illustration of this new species were based on collections deposited in the JBB herbarium of the Botanical Garden of Bogotá. Vegetative and inflorescence measurements were made on dry herbarium material, while the floral and fruit measurements were obtained from rehydrated herbarium material. The conservation status is suggested based on the IUCN criteria.

Key results: Miconia mahechae sp. nov. is characterized by the lianescent shrubby habit, oblong internodes, dense lanate indumentum of elongated moderately roughened trichomes on internodes, petioles and inflorescence, ovate to elliptical and 3-nerved leaves with obtuse to acute apex and smooth adaxial leaf surface, inconspicuous bracteoles, oblong-obovate anthers with an apical pore, and basal connective without appendages.

Conclusions: This new species is morphologically close to $M$. ruizteranii; however, the new species differs by the shape of its anthers and the indumentum of stems and inflorescences. Miconia mahechae has been collected only in areas surrounding the city of Bogotá and the Chingaza and Sumapaz National Parks, in areas of the high Andean forest. Based on the IUCN criteria, we suggest its categorization as Vulnerable (VU).

Key words: high Andean forest, Miconieae, Northern Andes, taxonomy.

${ }^{1}$ Herbario JBB, Jardín Botánico de Bogotá, Cl. 63 \#6895, Bogotá D.C., Colombia.

${ }^{2}$ Autor para la correspondencia: hummendoza@ gmail.com, hmendoza@jbb.gov.co
Recibido: 29 de septiembre de 2021

Revisado: 7 de noviembre de 2021.

Aceptado por Marie-Stéphanie Samain: 14 de diciembre de 2021.

Publicado Primero en línea: 19 de enero de 2022.

Publicado: Acta Botanica Mexicana 129 (2022)

(c) (7) Este es un artículo de acceso abierto bajo la licencia Creative Commons 4.0 Atribución-No Comercial (CC BY-NC 4.0 Internacional).
Citar como: Mendoza-Cifuentes, H. 2022. Una nueva especie de Miconia (Melastomataceae) de los bosques altoandinos de Bogotá D.C., Colombia. Acta Botanica Mexicana 129: e1979. DOI: https://doi. org/10.21829/abm129.2022.1979 


\section{Introducción}

Miconia Ruiz \& Pav., con cerca de 1900 especies, es el género de las angiospermas leñosas más diverso del neotrópico y uno de los más grandes del planeta (Goldenberg et al., 2008, 2013; Michelangeli et al., 2016, 2019). Colombia es el país con mayor diversidad del género, ya que alberga alrededor de 600 especies (Almeda et al., 2016), la mayor parte de ellas distribuidas en la región andina.

La reciente revisión de colecciones, en el marco de la realización de la monografía de Melastomataceae para la Flora de Bogotá, permitió el hallazgo de varias colectas con caracteres no concordantes con las especies de Miconia conocidas en Colombia. En este artículo se describe una nueva especie hasta ahora restringida a Bogotá D.C. y alrededores, con caracteres únicos de hábito de crecimiento y tipos de tricomas.

\section{Materiales y Métodos}

Se revisaron los ejemplares herborizados del herbario JBB del Jardín Botánico de Bogotá, Colombia. Para la descripción se realizaron medidas de las partes vegetativas e inflorescencias en material seco de herbario y utilizando un calibrador digital de $0.01 \mathrm{~mm}$ de precisión marca Mitutoyo (Serie 500, Neuss, Alemania). Las medidas de las partes florales se basaron en material seco rehidratado. Los tricomas se fotografiaron con una cámara MC190 HD (Wetzlar, Alemania) a partir material seco y posteriormente fueron clasificados con base en el atlas de tricomas de Melastomataceae de Wurdack (1986). Se sugiere su estado de conservación con base en el Área de Ocupación (AOO) y Extensión de Ocurrencia (EOO), utilizando para ello la herramienta GeoCat (Bachman y Moat, 2012) y aplicando las directrices y criterios de la IUCN (2017).

\section{Resultados}

Miconia mahechae Humberto Mend., sp. nov. Figs. 1, 2.

TIPO: COLOMBIA. Bogotá D.C., Sumapaz, corregimiento San Juan de Sumapaz, vereda San Antonio, camino hasta la casa de Saúl Chavarro, 3345 m, 46'54.4" N, 74¹9'35.6"W, 29.V.2018, fl, fr, F. Fajardo et al. 4327 (holotipo: JBB!, isotipos: COL!, UDBC!).
Miconia mahechae is distinguished by its lianescent shrubby habit, oblong internodes, dense lanate indument of elongated moderately roughened trichomes (type 17 of Wurdack, 1986) on internodes, petioles and inflorescences, ovate to elliptical and 3-nerved leaves with obtuse to acute apices and smooth adaxial leaf surfaces, inconspicuous bracteoles, oblong-obovate anthers with an apical pore and basal connectives without appendages or extensions. It is morphologically similar to $M$. ruizteranii Wurdack, but the latter is an erect shrub or small tree with acuminate leaves, rough blistered adaxial leaf surfaces, persistent and conspicuous bracteoles, truncate and 4-porate anthers, and shortly prolonged basal connectives.

Arbusto lianescente; entrenudos $1-4.3 \mathrm{~cm}$ de largo, oblongos; indumento sobre ramas, peciolo e inflorescencia denso lanosos, color crema o café claro, tricomas elongado-irregulares de superficie rugosa (tipo 17 de Wurdack, 1986), 0.4-1 mm de largo; hojas de un mismo nudo de tamaño similar, sin domacios; pecíolo $6-28 \mathrm{~mm}$ de largo, terete, ligeramente caniculado hacia el ápice; lámina 2.4$5 \times 1.3-3.4 \mathrm{~cm}$, ovada, anchamente ovada, oblongo-ovada o elíptica, margen espaciadamente denticulada o entera, base redondeada, obtusa o subcordada, ápice agudo a obtuso, haz lisa y glabra, envés con cubierta sobre las venas principales similar a los entrenudos, superficie glabra; nervadura con un par de venas secundarias acompañando a la vena media, basal o levemente plinervia, uniéndose a la vena primaria $2 \mathrm{~mm}$ arriba de la base; inflorescencia 4-7.5 $\mathrm{cm}$ de largo, terminal, paniculada y piramidal, con 30-120 flores pediceladas; pedúnculo $1.3-2 \mathrm{~cm}$ de largo; eje central con 4-7 nudos de ramificación; paracladios basales 2-4.5 cm de largo, con 2-3 niveles de ramificación; ápices de las ramas con múltiples flores siendo de un mismo punto (esciacioide); brácteas basales semejantes a hojas pequeñas; bracteolas inconspicuas; flor 6.2-6.5 mm de largo (longitud del hipanto más el estilo en flores abiertas sin incluir el pedicelo), 5-mera, diplostemona; pedicelos 1.2-1.7 mm de largo, con indumento denso de tricomas pinoides diminutos; hipanto 2.1-2.8 $\times$ 2.3-2.6 mm, cupuliforme, externamente con cubierta laxa de tricomas pinoides diminutos (tipo 25), internamente glabro; tálamo ca. $1.5 \mathrm{~mm}$ de largo; cáliz lobulado; tubo 0.3-0.33 mm de largo; lóbulo 0.3-0.6 × 


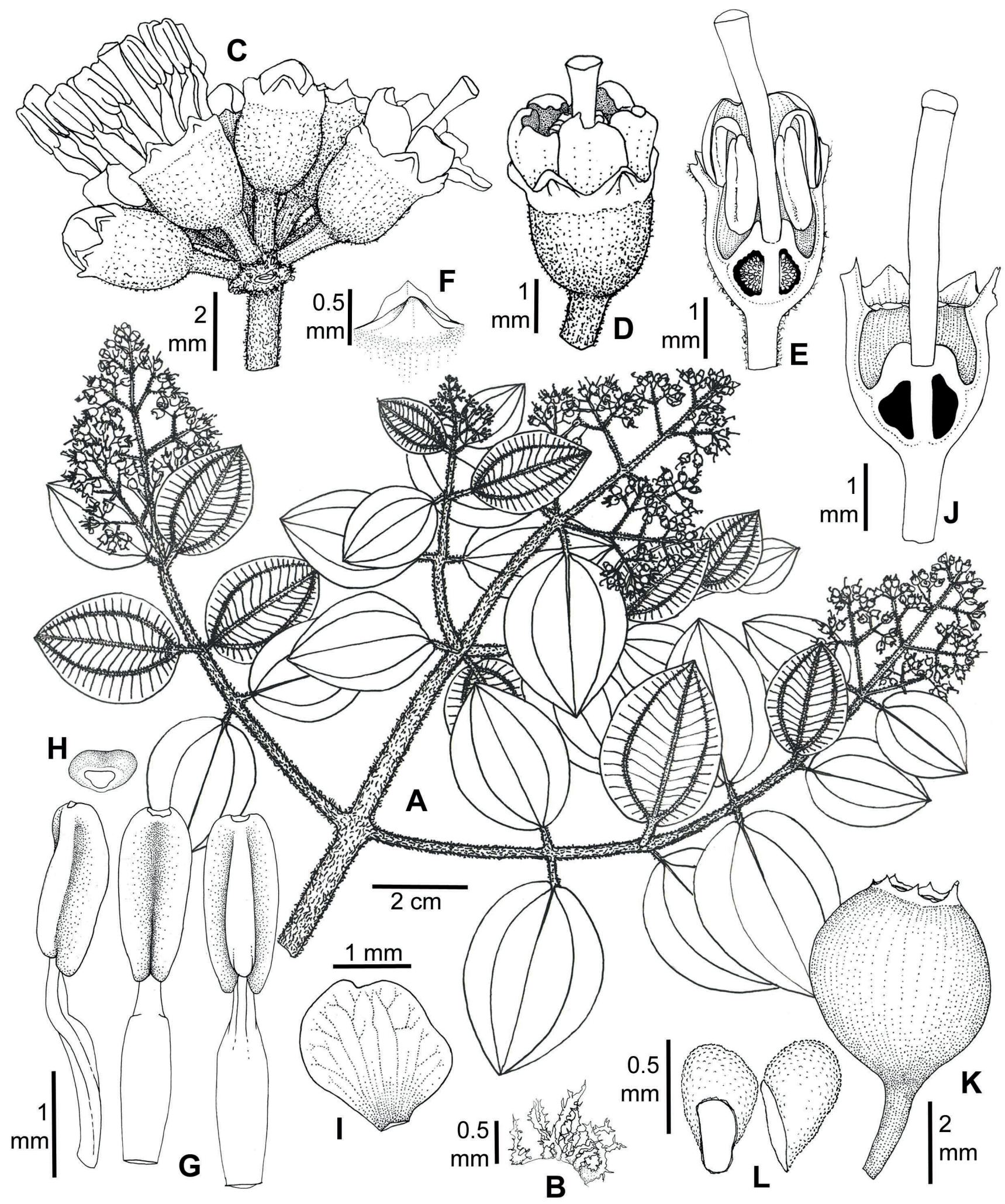

Figura 1: Miconia mahechae Humberto Mend. A. rama florífera; B. tricomas del entrenudo; C. ápice de las ramillas de la inflorescencia; D. flor en antesis; E. corte longitudinal de la flor en antesis; F. diente dorsal del cáliz; G. estambres en vista lateral (izquierda), ventral (centro), dorsal (derecha); H. ápice de la antera con poro; I. pétalo; J. corte longitudinal del hipanto-ovario; K. fruto; L. semillas. 
1.1-1.2 mm, anchamente triangular, membranoso; diente dorsal levemente desarrollado, 0.35-0.6 mm de alto; corola con los pétalos reflexos; pétalos 2-2.3 × 1.9-2.14 mm, anchamente obovados, apicalmente sigmoideo emarginados, glabros, blancos; estambres isomorfos, glabros, blancos; filamentos 2.2-2.3 mm de largo, 0.55-0.65 mm de ancho en su parte media; antera 1.9-2 × 0.7-0.9 mm, oblongoobovada, con un poro apical, 0.3-0.4 mm de ancho; conectivo dorsalmente engrosado, en la base con dos pequeños lóbulos ventrales, ca. $0.2 \mathrm{~mm}$ de largo; ovario $1 / 2$ ínfero, 3-locular, 1.5-2.1 mm de largo; parte basal fusionada al hipanto 0.9-1.2 mm, ápice libre del hipanto 0.82-0.84 mm, glabro; estilo levemente obcónico, recto, 4.3-5.1 mm de largo, glabro, exserto en etapas previas a la antesis de los estambres; estigma levemente expandido, 0.64-0.76 mm de diámetro; baya 5-6.5 mm de diámetro, con abundantes semillas, morado blancuzco cuando madura; semillas 0.6-0.7 $\mathrm{mm}$ de largo, ovoide; testa colliculada, brillantes, crema; rafe color café oscuro.

Distribución y hábitat: conocida solo del oriente y sur de Bogotá D.C., en la localidad de Sumapaz, y en los Parques Nacionales Naturales Chingaza y Sumapaz, entre 2950 y 3390 m de elevación (Fig. 2). Crece en zonas de bosque altoandino, en el interior del bosque en buen estado de conservación, pero también en los bordes.

Etimología: en honor al ingeniero forestal Gilberto Emilio Mahecha Vega (1942-2021), gran conocedor de la flora colombiana y quien realizó aportes significativos a las colecciones vivas del Jardín Botánico de Bogotá.

Fenología: los ejemplares con flores provienen de los meses de enero, febrero, mayo y junio; los que presentan frutos de febrero, mayo y junio.

Estado de conservación: esta especie tiene una EOO de ca. $487 \mathrm{~km}^{2}$, AOO de ca. $16 \mathrm{~km}^{2}$ y se conoce de siete localidades. Se registra para un área protegida, el Parque Nacional Natural (PNN) Chingaza, y se encuentra en la zona de amortiguación del PNN Sumapaz. Su área de distribución es pequeña, por lo que se podría categorizar como Amenazada (EN), bajo los criterios B2b(ii). No obstante, por su presencia en un área protegida se sugiere su categoría como Vulnerable (VU).

Ejemplares examinados (paratipos): COLOMBIA. Bogotá D.C., Sumapaz, corregimiento San Juan de Sumapaz, Las Vegas, 3390 m, 20.VI.2018, fl, fr, F. Fajardo et al. 4613 (JBB); Sumapaz, Nazareth, 3100 m, 15.Vl.2002, fr, M. Galvis et al. 213 (JBB); Sumapaz, vereda San Antonio, cuenca del río El Pilar, vía Sumapaz - San Bernardo, 3233 m, $4^{\circ} 06^{\prime} 29.77^{\prime \prime} \mathrm{N}, 74^{\circ} 19^{\prime} 54.96^{\prime \prime W}$, 28.VI.2021, fr, H. Mendoza et al. 22944 (JBB); Sumapaz, vereda San Antonio, cuenca del río El Pilar, vía Sumapaz - San Bernardo, 3103 m, $4^{\circ} 06^{\prime} 27.396^{\prime \prime} \mathrm{N}, 74^{\circ} 20^{\prime} 5.352^{\prime \prime W}$, 30.VI.2021, fr, H. Mendoza et al. 22951 (JBB). Cundinamarca, Fómeque, Parque Nacional Natural Chingaza, $3182 \mathrm{~m}, 4^{\circ} 35^{\prime} 51.4^{\prime \prime} \mathrm{N}, 73^{\circ} 43^{\prime} 10.7^{\prime \prime} \mathrm{W}$, 8.II.2014, fl, J. L. Contreras Herrera et al. 1746 (JBB); loc. cit., 08.II.2014, fr, C. Vargas et al. 2915 (JBB); Guasca, vereda Concepción, BioAndina, 2950 m, 446'21.8"N, 734' 40.8' 'W, 15.I.2015, fl, F. Henao-Díaz et al. 343 (JBB).

\section{Discusión}

Miconia mahechae se caracteriza por el hábito arbustivo-lianescente, el indumento denso-lanoso de tricomas alargados irregulares rugosos (tipo 17 de Wurdack, 1986) en ramas, peciolo e inflorescencia, los entrenudos oblongos, las hojas ovadas a elíptica 3-nervadas, con ápice no acuminado y la haz lisa, bractéolas inconspicuas, las anteras oblongo-obovadas con un poro apical, y el conectivo basal de la antera sin prolongaciones. La especie más semejante es $M$. ruizteranii de Venezuela. No obstante, esta última es un arbusto o arbolito erecto con cubierta café oscuro de tricomas pinoides, lámina foliar estrecho-ovada de ápice acuminado y haz ampollado-rugosa, flores con bractéolas conspicuas, inflorescencias laxas (vs. moderadamente congestionadas en $M$. mahechae), hipanto de $1.5 \mathrm{~mm}$ de largo (vs. 2.1-2.8 mm), pétalo $1.2 \mathrm{~mm}$ de largo (vs. 2-2.3 mm), filamento 1.8-1.9 mm de largo (vs. 2.2-2.3 mm), antera 0.6-0.7 $\mathrm{mm}$ de largo, obovado-truncada y abriendo en un boquete apical (vs. 1.9-2 mm, obovadas y con un pequeño poro apical), y el conectivo basal prolongado (vs. no prolongado) (Wurdack, 1972).

Otras especies con alguna semejanza en Colombia son Miconia cataractae Triana, la cual difiere por ser arbustos erectos con cubierta de tricomas dendríticos estipitados (Tria- 


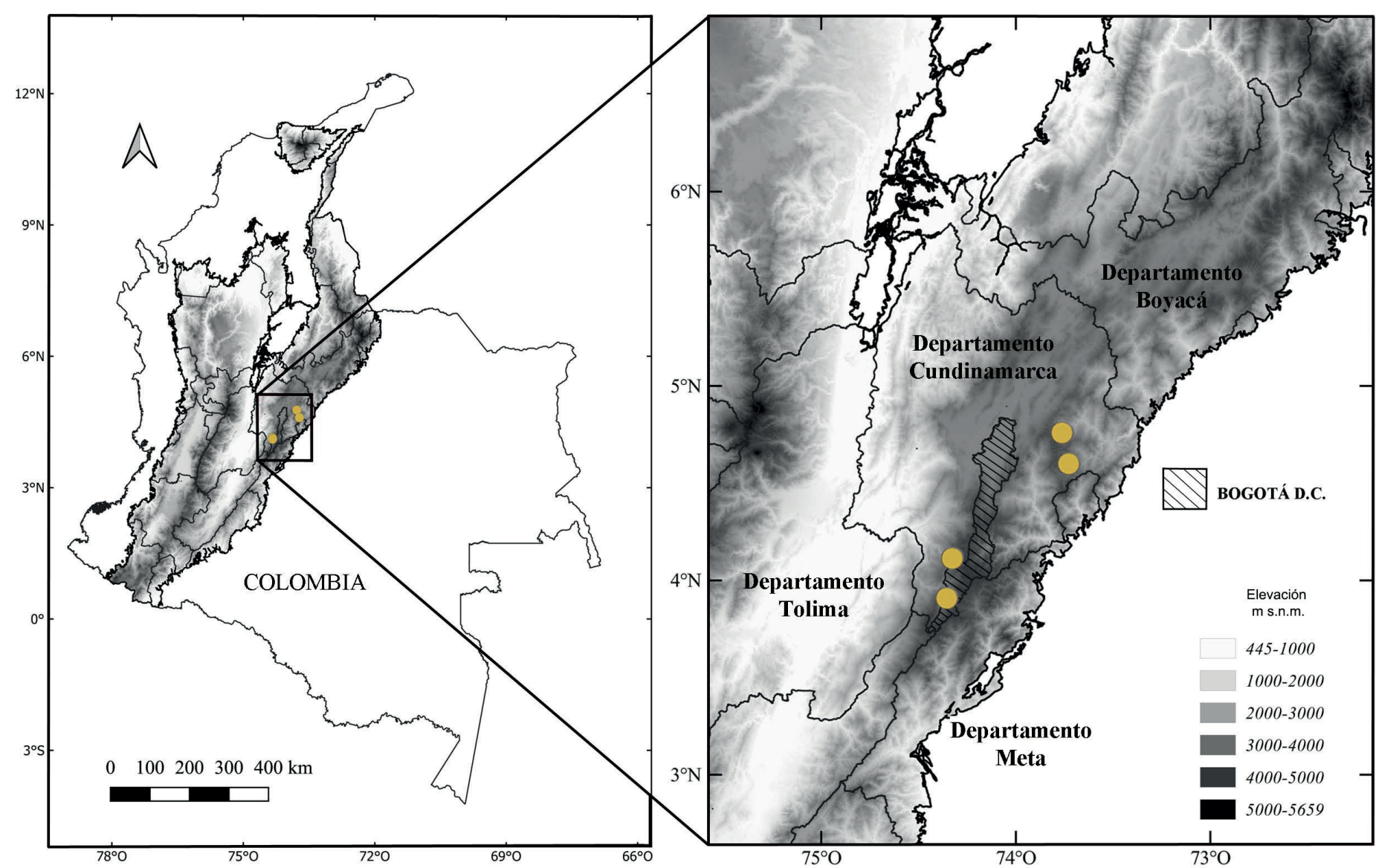

Figura 2: Distribución geográfica de Miconia mahechae Humberto Mend. (puntos amarillos), norte de los Andes, Colombia.

na, 1872), y M. elaeoides Naudin que difiere principalmente por ser arbustos erectos con cubierta de tricomas pinoides (Naudin, 1850). En Venezuela otra especie parecida es Miconia trujillensis Wurdack, pero esta última presenta tallos subcuadrangulares, cubierta de tricomas barbelados cortos, hojas 5-nervadas y glabras por el envés, y flores 4-meras (Wurdack, 1972). En Ecuador se encuentran con alguna semejanza M. pichinchensis Benth., la cual difiere en la cubierta de tricomas dendrítico-estrellados cortos, entrenudos subcuadrangulares, hojas acuminadas 5-nervadas y ovario 5-locular, así como $M$. bolivarensis Wurdack con la haz bullado-ampollada, cubierta de tricomas pinoides dendrítico-estrellados y hojas 5-nervadas (Bentham, 1845; Wurdack, 1973, 1980).

\section{Contribución de autores}

HMC concibió, adquirió los datos, revisó colecciones y escribió el manuscrito.

\section{Financiamiento}

Este estudio fue financiado por el Jardín Botánico de Bogotá, Subdirección Científica, Proyecto Flora de Bogotá.

\section{Agradecimientos}

Expreso sinceros agradecimientos al Jardín Botánico de Bogotá y al equipo de investigadores y técnicos del Herbario JBB. En especial a Boris Villanueva por el apoyo en la realización del proyecto Melastomataceae de la Flora de Bogotá y a los investigadores Andrés Orejuela y Carlos Vargas por correcciones al artículo y elaboración del mapa de distribución. Especial agradecimiento a Fabian A. Michelangeli y un revisor anónimo por sus valiosas recomendaciones.

\section{Literatura citada}

Almeda, F., M. Alvear, H. Mendoza-Cifuentes, D. S. Penneys y F. Michelangeli. 2016. Melastomataceae. In: Bernal, R., 
R. Gradstein y M. Celis (eds.). Catálogo de las Plantas de Colombia. Instituto de Ciencias Naturales, Universidad Nacional de Colombia y University of Göttingen. Bogotá, Colombia. Pp. 1585-1664.

Bachman, S. y J. Moat. 2012. GeoCAT an open source tool for rapid Red List assessments. http://geocat.kew.org (consultado diciembre de 2021).

Bentham, G. 1845. Plantas Hartwegianas imprimis Mexicanas. Publicado por el autor. London, UK. Pp. 182.

Goldenberg, R., D. S. Penneys, F. Almeda, W. S. Judd y F. A. Michelangeli. 2008. Phylogeny of Miconia (Melastomataceae): patterns of stamen diversification in a megadiverse neotropical genus. International Journal of Plant Sciences 169(7): 963-979. DOI: https://doi. org/10.1086/589697

Goldenberg, R., F. Almeda, M. K. Caddah, A. B. Martins, J. Meirelles, F. A. Michelangeli y M. Weiss. 2013. Nomenclator botanicus for the Neotropical genus Miconia (Melastomataceae: Miconieae). Phytotaxa 106(1): 1-171. DOI: http://doi. org/10.11646/phytotaxa.106.1.1

IUCN. 2017. Guidelines for using the International Union for Conservation of Nature Red list categories and criteria. Version 13. Prepared by the Standards and Petitions Subcommittee. http://www.iucnredlist.org/documents/ RedListGuidelines.pdf (consultado diciembre de 2021).

Michelangeli, F. A., R. Goldenberg, F. Almeda, W. S. Judd, E. R. Bécquer, G. Ocampo, G. M. Ionta, J. Jr. Dan Skean, L. C.
Majure y D. S. Penneys. 2019. Nomenclatural novelties in Miconia (Melastomataceae: Miconieae). Brittonia 72: 82121. DOI: https://doi.org/10.1007/s12228-018-9546-0

Michelangeli, F. A., F. Almeda, M. Alvear, E. R. Bécquer, J. Burke, M. K. Caddah, R. Goldenberg, G. M. Ionta, W. S. Judd, L. C. Majure, J. Meirelles, A. N. Nicolas, G. Ocampo, D. S. Penneys, J. Jr. Dan Skean y C. Ulloa-Ulloa. 2016. (2462) Proposal to conserve Miconia, nom. cons. against the additional names Maieta and Tococa (Melastomataceae: Miconieae). Taxon 65(4): 892-893. DOI: https://doi.org/10.12705/654.27

Naudin, C. V. 1850. Melastomacearum monographicae descriptionis. Annales des Sciences Naturelles, Botanique, sér. 3, 16(2): 223-224.

Triana, J. J. 1872. Les Mélastomacées. Transactions of the Linnean Society of London 28(1): 1-188. DOI: https://doi. org/10.1111/j.1096-3642.1871.tb00222.x

Wurdack, J. J. 1972. Certamen Melastomataceis XVIII. Phytologia 22(5): 413-414.

Wurdack, J. J. 1973. Certamen Melastomataceis XXI. Phytologia 26(1): 8-9.

Wurdack, J. J. 1980. Melastomataceae. In: Harling, G. y B. Sparre (eds.). Flora of Ecuador. No. 13. University of Göteborg, Riksmuseum. Stockholm, Sweden. Pp. 1-406.

Wurdack, J.J. 1986. Atlas of hairs for Neotropical Melastomataceae. Smithsonian Contributions to Botany 63: 1-80. DOI: https:// doi.org/10.5479/si.0081024X.63 